\title{
Dirac eigenmodes at the QCD Anderson transition
}

\author{
Matteo Giordano* \\ Institute for Nuclear Research of the Hungarian Academy of Sciences \\ Bem tér 18/c H-4026 Debrecen, Hungary \\ E-mail: kgteatomki.mta.hu
}

\section{Tamas G. Kovacs ${ }^{\star *}$}

Institute for Nuclear Research of the Hungarian Academy of Sciences

Bem tér 18/c H-4026 Debrecen, Hungary

E-mail: kgteatomki.mta.hu

\section{Ferenc Pittler}

MTA-ELTE Lattice Gauge Theory Research Group

Pázmány P. sétány 1/A H-1117 Budapest, Hungary

E-mail: pittlerabodri.elte.hu

\section{Laszlo Ujfalusi}

Department of Theoretical Physics, Budapest University of Technology and Economics H-1521, Budapest, Hungary

\section{Imre Varga}

Department of Theoretical Physics, Budapest University of Technology and Economics H-1521, Budapest, Hungary

\begin{abstract}
Recently we found an Anderson-type localization-delocalization transition in the QCD Dirac spectrum at high temperature. Using spectral statistics we obtained a critical exponent compatible with that of the corresponding Anderson model. Here we study the spatial structure of the eigenmodes both in the localized and the transition region. Based on previous studies in the Anderson model, at the critical point, the eigenmodes are expected to have a scale invariant multifractal structure. We verify the scale invariance of Dirac eigenmodes at the critical point.
\end{abstract}

The 32nd International Symposium on Lattice Field Theory,

23-28 June, 2014

Columbia University New York, NY

\footnotetext{
*Supported by the Hungarian Academy of Sciences under "Lendület" grant No. LP2011-011.
}

† Speaker. 


\section{Introduction}

It is well-known that the chiral cross-over of QCD is accompanied by a drastic change in the low-end of the spectrum of the Dirac operator. In a theory with massless quarks the spectral density at zero is proportional to the order parameter of the phase transition from the chirally broken to the symmetric phase [1]. Although in the real world even the lightest quarks are massive, most of the chiral symmetry breaking in QCD at low temperature still comes from spontaneous breaking. As a result, the qualitative picture of the Dirac spectrum across the transition is similar to that in the massless (chiral) limit. In Fig. 1 we show a cartoon of how the spectral density changes in QCD across the transition. At low temperature the spectral density at zero is finite, signaling the spontaneous breaking of chiral symmetry. At a point around the critical temperature of the crossover, the spectral density at zero vanishes and the low-end of the spectrum becomes more and more sparse as the temperature increases further.

These features of the Dirac spectrum are rather well-known. What is not so widely known, however, is that across the transition not only the spectral density but also the physical nature of the low eigenmodes changes considerably. Below the critical temperature $T_{c}$ the lowest modes are delocalized in the whole volume, no matter how large that is. In contrast, above $T_{c}$ the lowest part of the Dirac spectrum consists of modes localized on the scale of the inverse temperature [2]. However, even at high temperatures, the modes farther away from the edge, in the bulk of the spectrum, are still delocalized. In Fig. 1 the shaded regions indicate the localized modes, that are separated from the delocalized ones by the so called mobility edge. The localization properties of the eigenmodes is also reflected in the spectral statistics: eigenvalues corresponding to localized modes obey Poisson statistics, those corresponding to delocalized modes are described by WignerDyson statistics, known from random matrix theory, and widely used in the study of Dirac spectra below $T_{c}$.

The transition in the spectrum from localized to delocalized modes is analogous to Anderson transitions, first proposed to take place in conductors, in the presence of disorder in the crystal lattice [3]. In fact, already ten years ago it was suggested that the QCD chiral transition might be understood as an Anderson transition [4]. Later, using calculations in the instanton liquid model [5] and lattice QCD [6], qualitative support was obtained for this picture. Subsequently some of us studied Dirac spectra well above $T_{c}$. We found that the lowest part of the spectrum is always

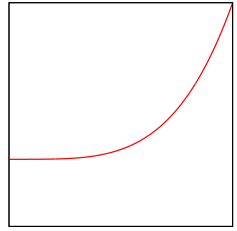

$T<T_{c}$

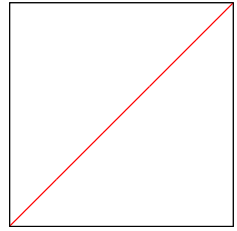

$T \approx T_{c}$

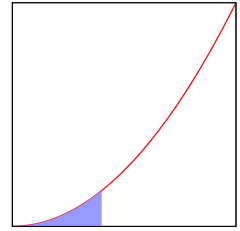

$T>T_{c}$

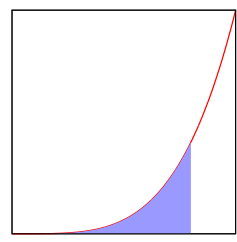

localized $\uparrow$ mobility edge

Figure 1: Schematic picture of the spectral density of the Dirac operator around zero as the system crosses the critical temperature from below. The temperature increases from left to right and the shaded areas indicate the localized modes at the low-end of the spectrum. 
localized and that the position of the mobility edge, separating localized and delocalized modes, is controlled by the temperature. Most importantly, the temperature where the mobility edge goes to zero and localized modes disappear, coincides with the chiral and deconfining cross-over temperature [2]. This lends further support to the conjecture that the Anderson-type transition in the spectrum and the chiral transition at $T_{c}$ are strongly related. In the present work, to elucidate this connection further, we propose to study the spatial structure of the Dirac eigenmodes across the transition. In particular, we present preliminary results supporting the expectation that at the transition the Dirac eigenmodes show signs of critical behavior similar to those found in Anderson transitions.

\section{Anderson transition in the Dirac spectrum above $T_{c}$}

The connection between Anderson transitions and the transition in the Dirac spectrum above $T_{c}$ is much more than a loose analogy. In previous work, using finite size scaling of the unfolded level spacing distribution, we showed that in the thermodynamic limit at the critical point $\lambda_{c}$ (the mobility edge) in the spectrum, spectral properties change in a non-analytic way. This also implies a diverging correlation length in the eigenmodes. Moreover, we found that the critical exponent $v$ characterizing this singularity is compatible with that of the three-dimensional unitary Anderson model [7]. This strongly suggests that above $T_{c}$ there is indeed a genuine Anderson transition from localized to delocalized modes in the Dirac spectrum and this transition is in the same universality class as that of the Anderson model.

However, we immediately have to point out that this "phase transition" in the spectrum does not imply a physical phase transition in QCD. This is because there is no physical control parameter that could tune the system to the critical point $\lambda_{c}$. The mobility edge $\lambda_{c}$ is just a point in the spectrum of the Dirac operator and thermodynamic quantities are averages over the whole Dirac spectrum. This has to be contrasted with the situation in Anderson transitions occurring in condensed matter systems. In that case the mobility edge is a genuine physical energy, a point in the spectrum of the one-electron Hamilton operator. By changing some physical control parameter (electron density, external field) the Fermi energy can be driven through the mobility edge. As the Fermi energy passes from the delocalized (conducting) states to the localized (non-conducting) states, the zero temperature conductivity changes non-analytically and the Anderson transition implies a genuine physical phase transition.

This is an important difference between the Anderson transitions in condensed matter systems and QCD. However, even in QCD there is a possibility for the Anderson transition to be accompanied by a real phase transition. This is at the temperature where the mobility edge reaches zero when the temperature is lowered from above $T_{c}$. In the thermodynamic limit, this is expected to happen at a well-defined temperature that we call $T_{\text {cAnd }}$. Note that $T_{\text {cAnd }}$ is well-defined even if the chiral transition is only a cross-over, like in QCD. To demonstrate how the mobility edge goes to zero, in Fig. 2 we plot the temperature dependence of the mobility edge in QCD with $N_{f}=2+1$ light staggered quark flavors with physical masses. We normalized the mobility edge by dividing it by the bare light quark mass to obtain a quantity that has a well-defined continuum limit. This also made it possible to plot data from simulations with different lattice spacings in the same figure. 


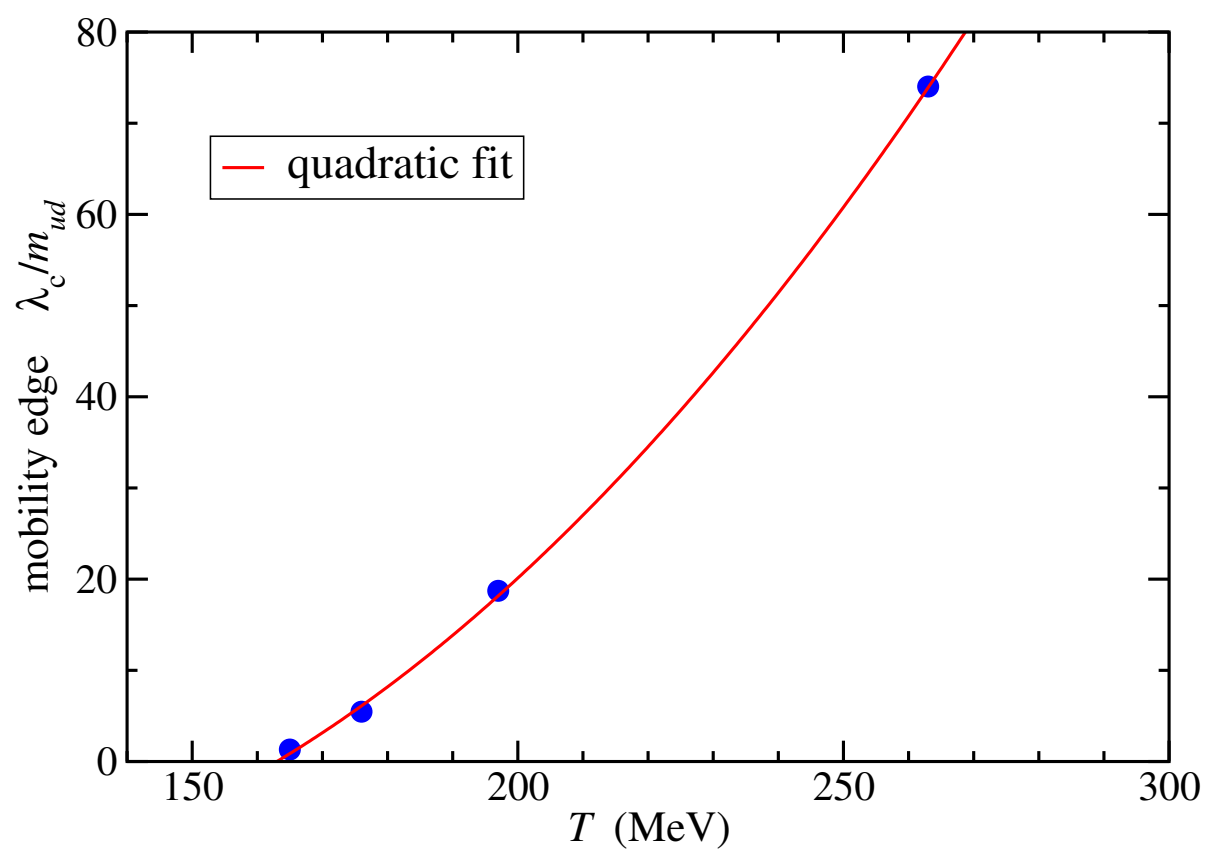

Figure 2: The temperature dependence of the mobility edge $\lambda_{c}$ obtained from lattice QCD simulations with $2+1$ flavors of staggered quarks at the physical point.

The line is a quadratic fit to the data. An extrapolation yields $T_{\text {cAnd }}=163(2) \mathrm{MeV}$, which is within the range of the chiral and deconfining cross-over [8].

We have seen that the finite temperature cross-over and the (dis)appearance of localized modes happen around the same temperature. In some QCD-like models with a genuine chiral phase transition not only $T_{\text {cAnd }}$ but $T_{c}$ is also well-defined and one can make a stronger statement. We found that in that case the two critical temperatures coincide (see Pittler's contribution at this conference [9]). It would be interesting to make connections between the physical quantities characterizing the critical behavior at the two transitions. To this end, we would need a detailed study around the point in the "phase diagram" where the $\lambda_{c}(T)$ critical line reaches the horizontal axis. The crucial question is whether the vanishing of the mobility edge is accompanied by an abrupt change in the spectral density in a finite interval around zero virtuality. If this happens and the change in the spectral density is non-analytic in the temperature, the theory has a phase transition.

\section{Dirac eigenmodes at the QCD Anderson transition}

Previously, most of our quantitative results about the QCD Anderson transition were based on statistical properties of the Dirac spectrum. However, it is well known in the case of Anderson transitions that the spatial structure of eigenmodes also encodes useful information about the transition. In the Anderson model, exactly at the critical point, the eigenmodes develop a peculiar multifractal structure [10]. Recently this has been exploited for a high precision determination of the critical exponent using finite size scaling of wave function observables [11]. 
Why is the structure of Dirac eigenmodes important in connection with the chiral transition? The reason is that any fermionic observable can be decomposed in terms of eigenmodes. In particular, the disconnected chiral susceptibility can be written in terms of eigenmode correlators of the form

$$
G(x)=\left\langle|\psi(0)|^{2}|\psi(x)|^{2}\right\rangle .
$$

If the eigenmodes have a (multi)fractal structure then the behavior of the correlator $G(x)$ is closely related to the fractal dimension(s) characterizing the eigenmodes [13]. This can provide a link between fractal properties of the critical eigenmodes and (pseudo)-critical properties of the chiral transition.

Before outlining our proposal for quantities to look at in connection with the eigenmodes, we have to explain some basic concepts concerning multifractals. Let us assume that $f$ is a function $f: \mathbb{R}^{d} \rightarrow \mathbb{R}$. Let us define the level sets of the function as

$$
S(a)=\{x: a<f(x)<a+\Delta a\} .
$$

If $f$ is a smooth function then the level sets either have dimension $d$ or are empty, depending on whether the given neighborhood of $a$ has an intersection with the range of $f$ or not. In contrast, if the dimension of the level sets $d(a)$ depends non-trivially on $a$, we call $f$ a multifractal weight function. We would like to study the average properties of a collection of such functions $f(x)=|\psi(x)|^{2}$ that are given only at the lattice sites and normalized to unity. We subdivide the lattice of linear size $L$ into smaller boxes of linear size $l \ll L$ and compute the coarse grained box weights

$$
\mu_{k}(l)=\sum_{\mathrm{x} \in \text { box } \mathrm{k}}|\psi(x)|^{2},
$$

where the sum runs over those lattice sites that are contained in box $k$. If the eigenmode has a multifractal structure, the system is scale invariant and consequently the distribution of box weights is expected to depend only on the ratio $l / L$ but not on $l$ and $L$ separately. If this is the case then the $l / L$ dependence of the distribution can be used to obtain the multifractal dimensions characterizing the eigenmodes [11].

As a first check, we would like to verify scale invariance at the mobility edge using a quantity derived from the box weights of eq. (3.3). For illustration, here we look at a quantity that has a transparent physical interpretation, called the information dimension. It is defined as

$$
\alpha_{1}=\frac{1}{\log (l / L)}\left\langle\sum_{k} \mu_{k} \log \left(\mu_{k}\right)\right\rangle
$$

where the sum runs over all the boxes of size $l$ and the averaging is performed for eigenmodes in a narrow spectral window on several gauge configurations. Since the eigenmodes are normalized, the quantity that is averaged is the information entropy of a given eigenmode corresponding to the given coarse graining box size $l$. It can be easily verified that for eigenmodes uniformly spread in the whole system volume, $\alpha_{1}$ is equal to the dimension of the system. On the other hand, for localized modes, the information entropy goes to zero if $L \rightarrow \infty$ and $l / L$ is kept fixed. This is because as the coarse graining box size becomes larger, eventually the whole eigenmode will be typically contained in one box. 


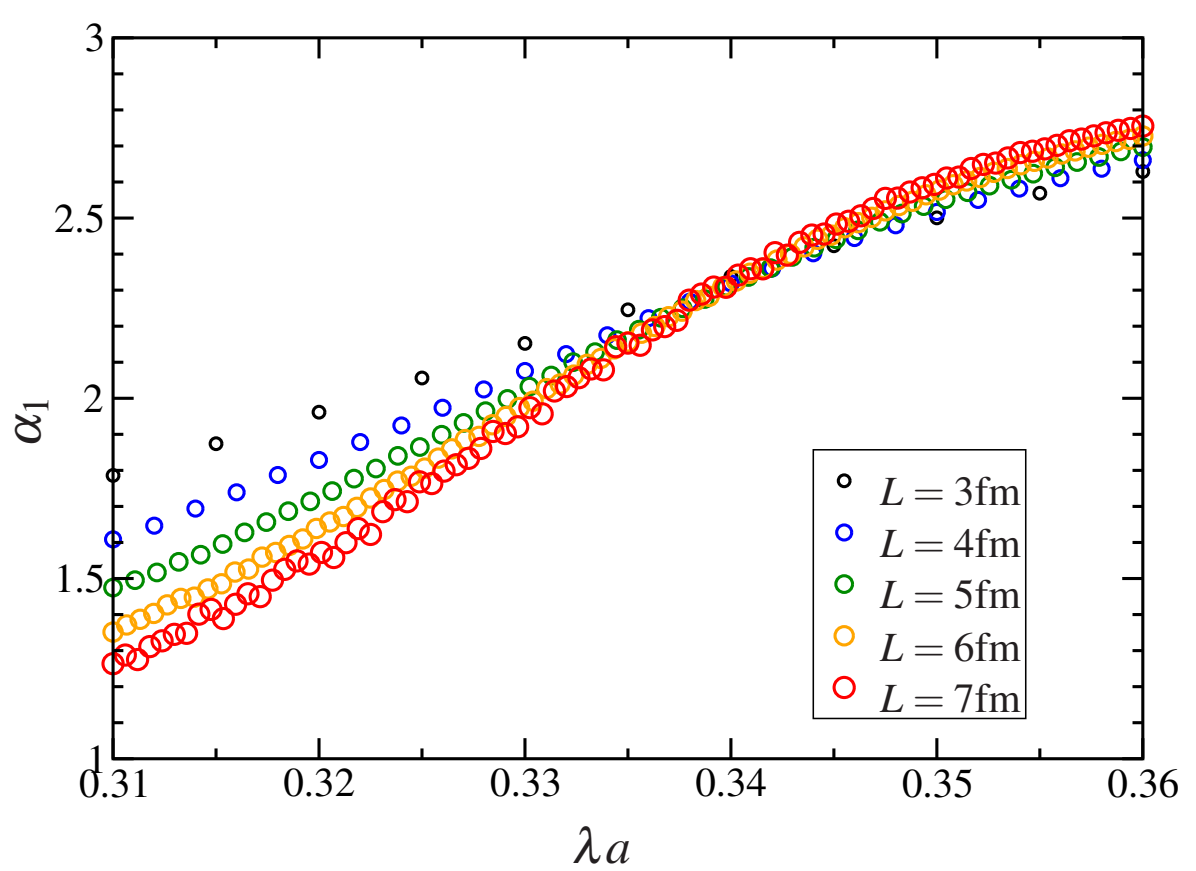

Figure 3: The information dimension $\alpha_{1}$ of eigenmodes as a function of the location in the spectrum. The average was computed separately for eigenmodes located in different narrow windows in the spectrum. The various symbols represent data taken in systems of different spatial sizes while keeping the ratio of coarse graining box size to the system size fixed at $l / L=0.125$.

The eigenmodes for the computation of $\alpha_{1}$ were obtained from lattice QCD simulations performed with the action of Ref. [12] at a fixed temperature well above $T_{c}$. We used spatial system sizes in the range $L=3-7 \mathrm{fm}$. In Fig. 3 we plot $\alpha_{1}$ as a function of the location of the eigenmodes in the spectrum. The different symbols correspond to different spatial system sizes and the coarse graining box size $l$ was always chosen such that the ratio $l / L$ was kept fixed at $l / L=0.125$. In the figure we can see that around $\lambda a=0.34$ the information dimension becomes independent of the system size, indicating that this is the critical point (mobility edge) in the spectrum where eigenmodes are scale invariant. It is reassuring that the critical point obtained here from eigenmode properties agrees with the one that we computed previously from spectral statistics [7].

Using quantities like the above described information dimension, the finite size scaling analysis of Ref. [7] can be repeated. This is work in progress but our preliminary results already indicate that both the critical point and the critical exponent we obtain are consistent with the previously determined values that were based on spectral statistics. However, it is interesting to note that the uncertainty of the results based on eigenmode observables is larger than those obtained from spectral statistics using the same ensemble of lattice configurations. This is because scaling violations, due to operators irrelevant in the RG sense, appear to be more sizeable for eigenmode observables than for observables derived from spectral statistics. For better precision one might need to consider systems of larger spatial sizes. 


\section{Conclusions}

Previously we found an Anderson-type localization-delocalization transition in the spectrum of the QCD Dirac operator at high temperature. Based on a finite size scaling analysis of spectral observables we obtained strong evidence that the transition is in the same universality class as that of the corresponding ( $3 \mathrm{~d}$ unitary) Anderson model. In the present paper we proposed a quantitative study of the spatial structure of the corresponding eigenmodes. This could provide further information concerning the nature of the transition. As a first consistency check we verified that at the critical point in the spectrum the coarse grained eigenmode box probabilities become scale invariant, as expected in Anderson transitions. We found that the critical point obtained from the scale invariance of the eigenmodes coincides with the one previously determined from spectral observables. Preliminary results of a finite size scaling analysis of eigenmode data indicate that the resulting critical exponent is also consistent with the one obtained from spectral data. This shows that in QCD, spectral and eigenmode properties signal the Anderson transition in a consistent way, exactly like in the Anderson model. It would be also interesting to determine the dimensions characterizing the multifractal eigenmodes at the critical point and compare those to results from the Anderson model. To this end we intend to study the dependence of the box probabilities on the ratio of the box size and the system size $(l / L)$. In the present study this quantity was kept fixed.

\section{References}

[1] T. Banks and A. Casher, Nucl. Phys. B 169, 103 (1980).

[2] T. G. Kovacs and F. Pittler, Phys. Rev. D 86, 114515 (2012) [arXiv:1208.3475 [hep-lat]].

[3] P. W. Anderson, Phys. Rev. 109, 1492 (1958).

[4] A. M. Garcia-Garcia and K. Takahashi, Nucl. Phys. B 700, 361 (2004) [cond-mat/0403557].

[5] A. M. Garcia-Garcia and J. C. Osborn, Nucl. Phys. A 770, 141 (2006) [hep-lat/0512025].

[6] A. M. Garcia-Garcia and J. C. Osborn, Phys. Rev. D 75, 034503 (2007) [hep-lat/0611019].

[7] M. Giordano, T. G. Kovacs and F. Pittler, Phys. Rev. Lett. 112, no. 10, 102002 (2014) [arXiv:1312.1179 [hep-lat]].

[8] S. Borsanyi et al. [Wuppertal-Budapest Collaboration], JHEP 1009, 073 (2010) [arXiv:1005.3508 [hep-lat]].

[9] M. Giordano, S. D. Katz, T. G. Kovacs and F. Pittler, PoS LATTICE 2014, 214 (2014).

[10] F. Evers and A. D. Mirlin, Rev. Mod. Phys. 80, 1355 (2008).

[11] A. Rodriguez, L. J. Vasquez, K. Slevin and R. Römer, Phys. Rev. Lett. 105, 046403 (2010); Phys. Rev. B 84, 134209 (2011).

[12] Y. Aoki, Z. Fodor, S. D. Katz and K. K. Szabo, JHEP 0601, 089 (2006) [hep-lat/0510084].

[13] T. Nakayama and K. Yakubo, "Fractal concepts in condensed matter physics", 1st ed. Springer Verlag (2003), p. 12, 48-49. 